\title{
Distributed Power Control for Underwater Acoustic Networks
}

\author{
Josep Miquel Jornet \\ Massachusetts Institute of Technology \\ Email: jmjornet@mit.edu
}

\author{
Milica Stojanovic \\ Massachusetts Institute of Technology \\ Email: millitsa@mit.edu
}

\begin{abstract}
Multi-hop transmission is considered for large coverage area in bandwidth-limited underwater acoustic networks. Discrete power control is introduced as a practical means of optimizing the overall system performance across the physical and the medium access control layers. The required number of power levels and the way in which they are distributed for increasing network densities is analyzed in light of minimum energy per bit consumption.

The system performance is evaluated for different frequency allocation patterns (center frequency $f_{c}$ and bandwidth $B$ ). The results show that the total energy per bit consumption can be reduced by moving to higher frequencies, where the background interference is decreased and a greater bandwidth is available. A greater bandwidth supports transmission at higher bit-rates, resulting in a twofold effect: first, the total energy consumption is reduced because the transmission time is shorter, and second, shorter packets are less likely to collide. These facts encourage the use of high bit-rates even if the application does not require it. In addition, they motivate a review of the medium access control protocols, whose performance depends on the number of collisions.

Two MAC protocols are considered: the Distance Aware Collision Avoidance Protocol (DACAP), a virtual carrier-sense-like protocol that completely avoids harmful collisions, and the simple Carrier Sensing ALOHA. Coupled with power control, both protocols are shown to be well-suited for networks containing static and mobile nodes which are not synchronized to a global clock.
\end{abstract}

\section{INTRODUCTION}

Multi-hopping is a well-established transmission technique in wireless communication systems. This concept is usually related to high density sensor or ad hoc networks, in which low-cost battery-powered nodes should minimize energy consumption without compromising the network connectivity and the ability to deliver data to a final destination. In underwater acoustic networks, multi-hopping offers not only the benefits of power savings, but also the possibility to utilize a greater data rate. This property is a consequence of the fact that the useful acoustic bandwidth depends on the transmission distance, increasing as the distance shortens [1]. The capacity of an acoustic relay link thus increases with the number of hops used to span a given distance [2].

The analysis presented in [2] is obtained for a noise-limited scenario, i.e. it does not take into account the presence of interference. As such, it serves as an upper bound on practical systems in which the channel access must be regulated, either in a deterministic or a random fashion. The capacity of a cellular underwater network where multiple access is regulated either by TDMA or FDMA was assessed in [3]. The effects of interference on the system capacity in a contentionbased acoustic network have been addressed in [4], showing similar results. This study shows that capacity follows the same trend in an interference-limited scenario as in the noiselimited one. In [5], the design of minimum energy routes is investigated, showing that in dense networks, there exists an optimal number of hops over which the system performance does not improve.

Power control for wireless networks has been widely addressed in the literature. A good review can be found in [6], where the impact of variable-range transmission power control on the network connectivity is investigated. Different ways of adapting the transmission power have been proposed, e.g. in accordance with the packet length [7], the average number of neighbors [8], or the measured link quality [9]. These methods show that the use of power control can improve the system performance in terms of energy consumption and network capacity.

In this paper, we focus on random channel access for underwater acoustic networks, addressing the design of discrete power control in light of minimum energy per bit consumption. Two MAC protocols are considered to verify the design: the simple Carrier Sensing ALOHA (CS-ALOHA), and a recently proposed virtual carrier sensing method, the Distance Aware Collision Avoidance Protocol (DACAP) [10]. In a multi-hop scenario, the performance of both protocols depends on the transmission power. Simply stated, too little power may lead to a loss of connectivity, while too much power causes unnecessary interference which prolongs the contention phase. The increasing levels of interference cause repeated transmissions with CS-ALOHA, thus increasing the overall energy consumption. With DACAP, collisions are avoided, but longer waiting times become necessary in the presence of stronger interference.

The paper is organized as follows. In Sec.II, underwater channel characteristics are reviewed. The power control algorithm and its parameters are defined in Sec.III. Integration of power control into the MAC protocols is discussed in Sec.IV. Sec.V is devoted to analyzing the system performance through simulation examples, and studying the impact of different power control parameters and frequency allocation. Conclusions are summarized in Sec.VI. 


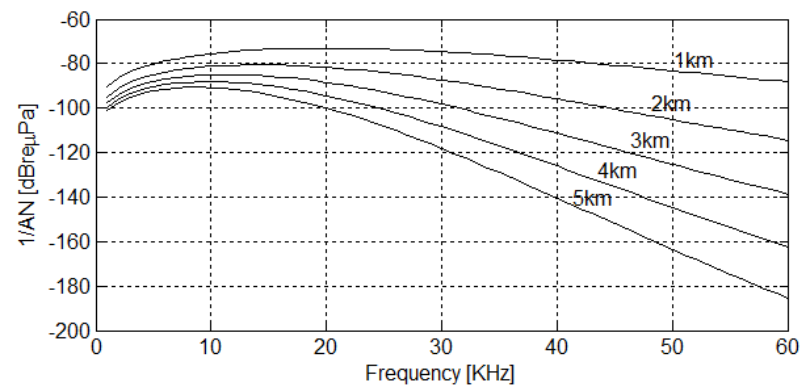

Fig. 1. Frequency-dependent part of the narrow-band SNR, $1 / A(l, f) N(f)$, for different transmission distances (spreading factor $k=1.5$ ).

\section{Review OF THE ACOUSTIC CHANNEL}

The acoustic attenuation, expressed in $\mathrm{dB}$, is given by

$$
10 \log A(l, f)=k \cdot 10 \log l+l \cdot 10 \log a(f)
$$

where $l$ and $f$ are the distance and frequency, respectively; $k$ denotes the spreading factor, and $a(f)$ is the absorption coefficient, which can be expressed empirically using Thorp's formula [1].

The ambient noise is approximated in the frequency range of interest by a power spectral density (p.s.d.)

$$
10 \log N(f) \approx \eta_{0}-18 \log f
$$

where $f$ is in $\mathrm{kHz}$, and the constant level $\eta_{0}$ is taken to be $50 \mathrm{~dB}$ for the quiet deep sea [1]. The narrow-band signal to noise ratio $(\mathrm{SNR})$ is given by

$$
S N R(l, f)=\frac{S(f) \Delta f / A(l, f)}{N(f) \Delta f}=\frac{S(f)}{A(l, f) N(f)}
$$

where $S(f)$ is the power spectral density of the transmitted signal and $\Delta f$ is a narrow frequency band around $f$. The factor $1 / A(l, f) N(f)$ is illustrated in Fig.1. For each transmission distance $l$, there clearly exists an optimal frequency $f_{o}(l)$ for which the maximal narrow-band SNR is obtained. Note that this result is invariant to shifting the noise p.s.d. $\eta_{0}$ by a fixed margin, which is done in practice to account for additional site-speific noise.

We define the $3 \mathrm{~dB}$ bandwidth $B_{3 d B}(l)$ as the range of frequencies around $f_{o}(l)$ for which $A(l, f) N(f)<$ $2 A\left(l, f_{o}(l)\right) N\left(f_{o}(l)\right)$. The optimal frequency $f_{o}(l)$ and its corresponding $B_{3 d B}(l)$ as a function of the transmission distance $l$ are plotted in Fig.2. The center frequency $f_{c}(l)$ and the ratio of bandwidth to center-frequency are also included. As the transmission distance is reduced, the optimal frequency is higher, as well as its corresponding $3 \mathrm{~dB}$ bandwidth.

Assuming that the transmitted signal p.s.d. is flat across the $3 \mathrm{~dB}$ bandwidth, the transmission power necessary to provide a target $\mathrm{SNR}, \mathrm{SNR}_{0}$, at a distance $l$ from the source is determined as

$$
P(l)=S N R_{0} B_{3 d B}(l) \frac{\int_{B_{3 d B}(l)} N(f) d f}{\int_{B_{3 d B}(l)} A^{-1}(l, f) d f}
$$
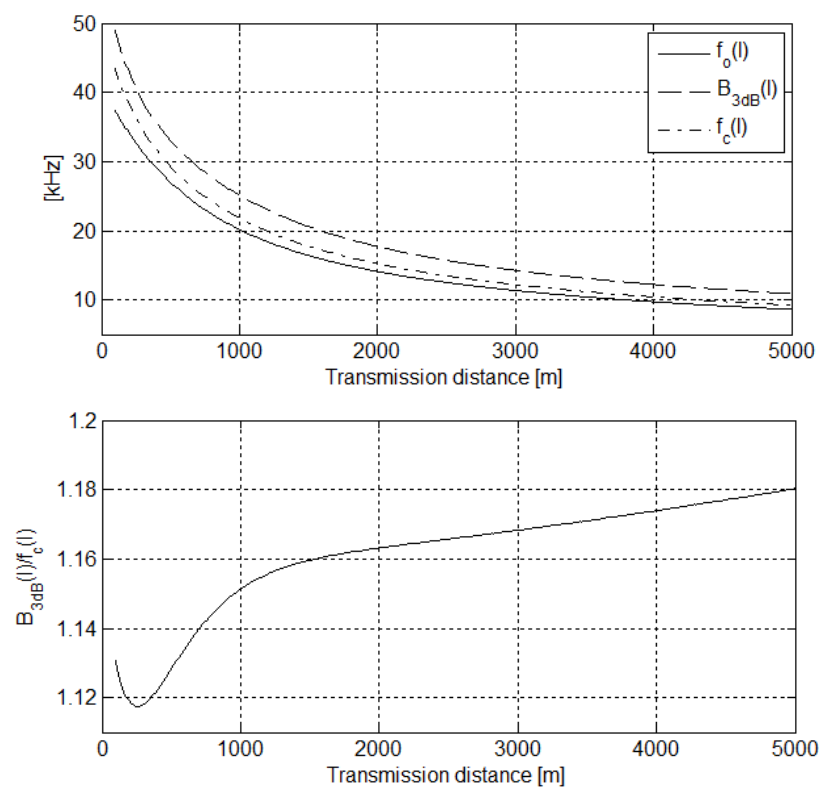

Fig. 2. Top: Optimal frequency $f_{0}(l), 3 \mathrm{~dB}$ bandwidth $B_{3 d B}(l)$, and center frequency $f_{c}(l)$. Bottom: bandwidth/center-frequency ratio as a function of transmission distance $l$ (spreading factor $k=1.5$ ).

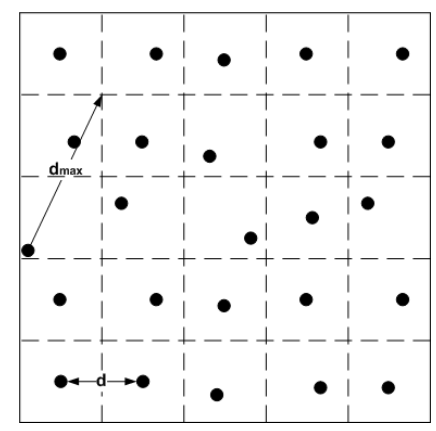

(a)

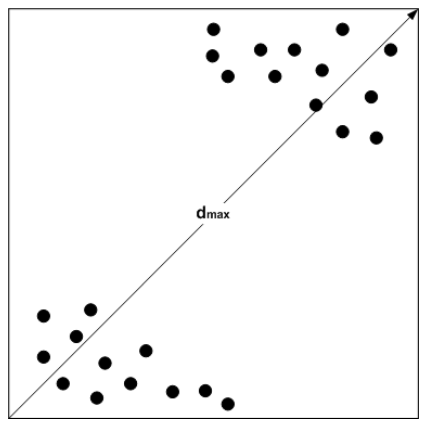

(b)
Fig. 3. Two possible scenarios: a) random location of the nodes within a grid and b) completely random location of the nodes.

\section{Power CONTROL}

We look at an underwater acoustic network containing both static and mobile nodes. The nodes are able to switch their transmission power $P$ over a finite set of power levels, ranging from some minimum to maximum, $P_{0}, P_{1}, \ldots, P_{N-1}$.

\section{A. Network topology and maximal power}

We define the maximal transmission power as the minimum that still guarantees connectivity between any two nodes in the network. Two nodes are said to be physically connected if they can reach each other with a target SNR. In light of multi-hop communications, two nodes are virtually connected if there exists at least one path of physically connected nodes between them.

Fig.3 illustrates two examples of random node placement in a network. For simplicity, we restrict our attention to a two-dimensional geometry, keeping in mind that extension to 


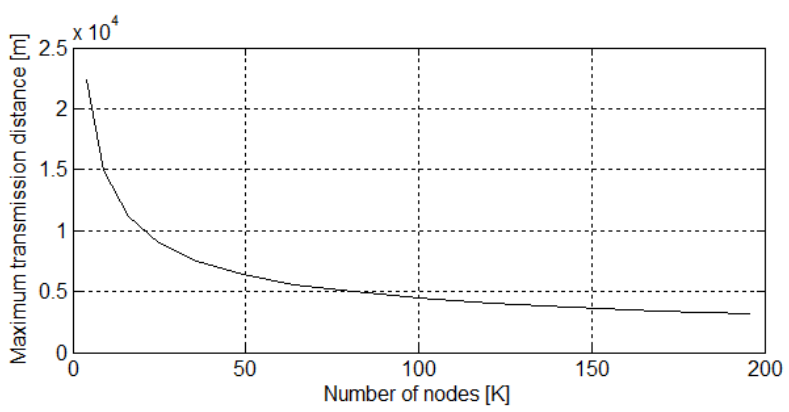

Fig. 4. Maximal transmission distance as a function of the number of nodes deployed over a fixed area $\mathrm{S}=400 \mathrm{~km}^{2}$ (6).

three-dimensions is straightforward. We focus on the scenario presented in Fig.3a. In this case, the area over which the network is deployed can be envisioned as a grid, where each square contains one node randomly placed within it. The network node density is given by

$$
\rho=\frac{K}{S}=\frac{1}{d^{2}}
$$

where $S$ is the network area, $K$ is the total number of nodes in it, and $d$ is the average inter-node distance. The maximal power $P_{N-1}$ is associated with a transmission distance

$$
d_{\text {max }}=\sqrt{5} d=\sqrt{\frac{5}{\rho}}
$$

For a fixed area $S=400 \mathrm{~km}^{2}$, the maximal transmission range as a function of the number of nodes is shown in Fig.4.

There are other situations which may be of interest. For example, the $K$ nodes can be randomly placed over the entire area $S$ as shown in Fig.3b. In this case, the maximum transmission power does not scale with the network node density. The worst case, illustrated in the figure, implies that the maximum transmission distance can be much greater than the one given in (6).

In what follows we will assume the first network topology shown in Fig.3a, as one that is more likely to occur in practice. If this topology is violated, i.e. two nodes become separated by more than $d_{\max }$ given in (6), some parts of the network will lose connectivity. However, in a network that contains mobile nodes, this situation may only be temporary.

\section{B. Step size between power levels}

Assuming a uniform separation of power levels in $\mathrm{dB}$, the step size $\Delta$ between two consecutive levels is defined by

$$
P_{0}=P\left(d_{0}\right), P_{n}=\Delta^{n} P_{0}=P\left(d_{n}\right), P_{N-1}=P\left(d_{\max }\right)
$$

where $d_{n}$ is the distance corresponding to the power $P_{n}$, i.e. the two are related by the expression (4). Alternatively, the separation between two consecutive levels can be defined in terms of a uniform increase in the coverage distance, $\delta$ :

$$
P_{0}=P\left(d_{0}\right), P_{n}=P\left(d_{0}+n \delta\right), P_{N-1}=P\left(d_{\max }\right)
$$

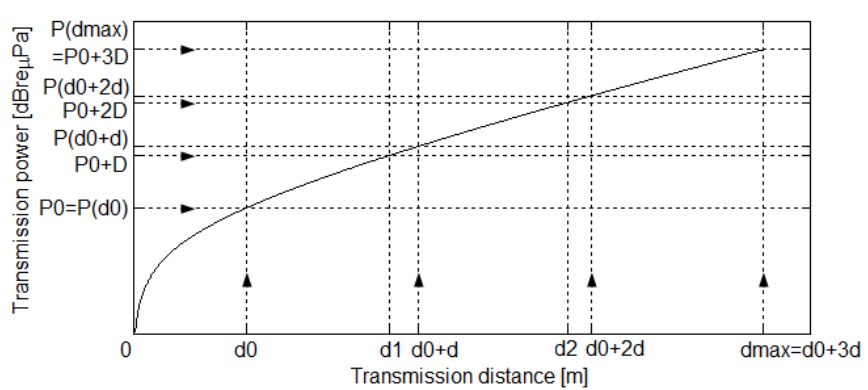

Fig. 5. Power levels and corresponding distances for the two strategies (7) and (8), using a step size of $\Delta=14 \mathrm{~dB}$ and $\delta=765 \mathrm{~m}$, respectively. The network has 64 nodes deployed over $100 \mathrm{~km}^{2}$ in a grid-uniform manner. $d_{0}=500 \mathrm{~m}$, $d_{\max }=2795 \mathrm{~m}, f_{c}=40 \mathrm{kHz}, B=30 \mathrm{kHz}, \mathrm{SNR}_{0}=20 \mathrm{~dB}$.

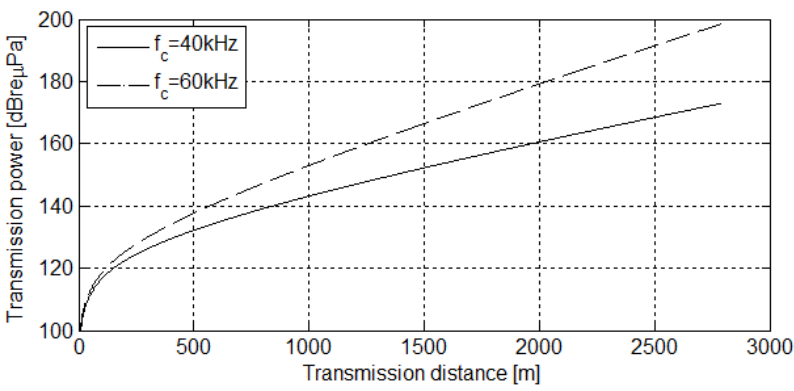

Fig. 6. Transmission power as a function of distance for two center frequencies. $B=30 \mathrm{kHz}, \mathrm{SNR}_{0}=20 \mathrm{~dB}$.

The step size is defined in $\mathrm{dB}$ as

$$
10 \log \Delta=\left(10 \log P\left(d_{\max }\right)-10 \log P\left(d_{0}\right)\right) /(N-1)
$$

and in meters as

$$
\delta=\left(d_{\max }-d_{0}\right) /(N-1)
$$

Fig.5 shows the two power distribution patterns when using $N=4$ levels, in a network with 64 nodes deployed over $100 \mathrm{~km}^{2}$. In this example, the differences between the two power allocation patterns are small, i.e. $P\left(d_{n}\right) \approx P\left(d_{0}+n \delta\right)$.

In Fig.6, the transmission power is plotted versus distance for two different center frequencies. Both curves show a nearly constant slope for the range of distances considered, which confirms the above observation. Due to the convenience of defining the power levels in terms of a uniform increase in distance, we will use this definition in what follows.

\section{Number of power levels}

The last parameter required to completely characterize the power control is the number of power levels $N$. Increasing the number of levels allows finer tuning of the power; however, a small number of levels is of interest to practical implementation. We conjecture that there is an effect of diminishing returns in energy savings when it comes to increasing the number of levels beyond some point. We will assess this issue through numerical simulation in Sec.V-B. 


\section{Medium Access Control}

Two MAC protocols are considered: the simple carrier sensing variation of the 1-persistent ALOHA, and the Distance Aware Collision Avoidance Protocol [10].

\section{A. Overview of CS-ALOHA}

A node using CS-ALOHA listens to the channel before transmitting, and if it finds the channel idle, it begins to transmit data. Taking into account the half-duplex operation of current acoustic modems, a sender cannot detect collisions while transmitting, and will always transmit an entire data packet. It will deduce that its transmission has collided if a positive acknowledgment has not arrived after a certain waiting time. In that situation, it will retransmit according to the same procedure, unless the maximum number of retransmission attempts has been exhausted.

CS-ALOHA stands out for its simplicity and average end-toend delay performance, but it is not the best option in terms of energy consumption, due to the energy lost in retransmissions.

\section{B. Overview of DACAP}

DACAP is a virtual carrier-sense-like MAC protocol that has recently been proposed for underwater networks [10]. It implements an exchange of short control packets for avoiding data packet collisions, thus maximizing the network throughput.

The protocol is based on the following steps:

- Upon receiving a request to send (RTS), a node sends a clear to send (CTS), and waits for a data packet. If during the waiting time another RTS is overheard, the node sends a short warning to its partner.

- Upon receiving a CTS, the transmitter waits for those nodes whose attempts to transmit may result in collisions. If during this time another CTS is overheard or a warning packet arrives, the transmission is deferred by a random back-off time. Otherwise, the transmission of the data packet proceeds.

DACAP was shown to improve the system performance in terms of energy per bit consumption, at the expense of increasing the average end-to-end delay due to postponements.

\section{Integration of power control}

When using discrete power control, the routing protocol, the medium access control, and the physical layer functionalities are tightly coupled. By choosing the path, a routing protocol decides which power level should be used. The medium access control should then adapt specific parameters, such as waiting, or back-off times, according to the new transmission distance. Finally, switching the transmission power to a new level occurs at the physical layer.

In our simulations, we assume that the network topology is known, i.e. all nodes know all others' positions. Then, routes are geographically pre-established using Dijkstra's algorithm, in which the cost between two nodes is defined as the power level required to guarantee physical connectivity between them. The target SNR for physical connectivity is set to
$\mathrm{SNR}_{0}=20 \mathrm{~dB}$ in our simulations. A node will tune its power to the minimal level required to reach the next node along the packet route.

A more general approach, in which each transmitting node only needs its current position and the position of the final destination, is deferred to a later publication [12].

\section{Implicit Acknowledgment}

Apart from the end-to-end acknowledgment which may be generated at the transport or application layer, each intermediate node expects a positive acknowledgment from the current receiver. If nodes use omni-directional transducers, the transmitter can deduce that the last data transaction has been properly completed if it overhears its own packet being relayed. However, this may not always be possible. If the power level used to reach the next node is lower than the one used previously, the acknowledgment should be sent explicitly using a higher power level. An explicit acknowledgment should also be sent by the final destination when the data packet reaches it.

\section{Performance Analysis}

To verify the concepts introduced, we have used a discreteevent underwater acoustic network simulator implemented in standard Python [13].

The simulation scenario corresponds to the one shown in Fig.3a, in which the maximum transmission range scales with the node density. The network is composed of four active nodes, a common sink in the center, and a varying number of relay nodes. A Poisson distribution with an average packet generation rate $\lambda=1$ packet/min is assumed. Each packet contains a fixed number of bits, 9600 in our simulation.

The system performance is measured in terms of average energy per bit consumption. The energy invested in transmission, listening, and active reception of control and data packets, as well as their possible retransmissions, is taken into account. For a given transmission distance $d_{n}$, the transmission energy per bit is given by

$$
E_{b, n}=\frac{P_{n}}{\alpha B}
$$

where $\alpha$ stands for the bandwidth efficiency and it is assumed to be constant and equal to $1 \mathrm{bit} / \mathrm{Hertz}$, and $B$ is the bandwidth. We will look at two cases, one in which the bandwidth is fixed, i.e. set a-priori irrespective of the transmission distance, and another in which it is determined as the $3 \mathrm{~dB}$ bandwidth corresponding to the average transmission distance. The power required for listening is set to $1 \mathrm{~mW}$, and the power required for active reception (demodulation and detection of a data packet) is $80 \mathrm{~mW}$. In addition to the energy per bit, the total number of collisions, as well as the average packet end-to-end delay are also measured to illustrate the performance.

Before focusing on the effects of different power distribution schemes and frequency allocation patterns, the benefits of increasing the relay density are analyzed. 


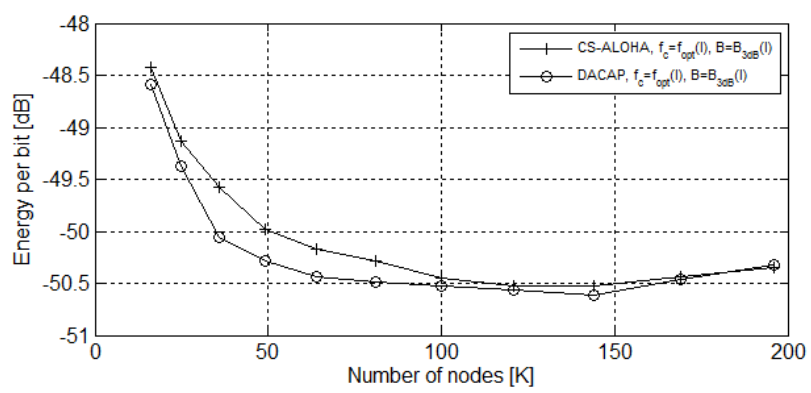

Fig. 7. Energy per bit consumption for CS-ALOHA and DACAP using power control with 4 uniformly distributed levels. A fixed number of informationgenerating nodes (four) and a varying number of relay nodes are deployed over $20 \mathrm{~km} \times 20 \mathrm{~km}$ area.

\section{A. Effects of relay density}

Fig.7 shows the average energy per bit consumption as the number of relays is varied. For each node density, $d_{\max }$ is obtained from (6). The system center frequency and the available bandwidth are determined according to the principles of in Sec.II, as $f_{c}(d)$ and $B_{3 d B}(d)$, where $d$ is the average internode distance. The number of available power levels is $N=4$.

As the number of nodes increases, using this frequency allocation pattern the average energy per bit consumption is reduced. This is due not only to the fact that the transmission power is lower, but also to the fact that the bandwidth available to shorter links is greater. Hence, a data packet containing the same number of bits takes less time to transmit at a higher bit rate. We also observe that there is a density of relay nodes above which there is no additional improvement in the system performance in terms of energy per bit consumption. This effect is related to the energy consumed for active reception of the transmitted packets. When multi-hopping, the transmission power can clearly be reduced, but the receiving power remains the same.

\section{B. Effect of the number of power levels $N$}

As the number of relay nodes increases, the energy consumption reduces mainly because the maximum transmission distance scales with the network density as (6). Therefore, even for a constant transmission power, i.e $N=1$, the energy consumption is clearly reduced.

Using more than one power level allows the system to allocate the power in a more efficient manner. Only those nodes that require the highest transmission power will use it, while all other nodes can use lower levels. By increasing the number of power levels, the power can be adjusted more accurately, reducing the total power consumption, as well as interference. However, having to use too many power levels may not be practical, and the question remains as to whether there is some number of levels that reaches a good compromise between energy efficiency and implementation complexity.

Fig.8 shows the average energy per bit consumption as a function of the number of nodes for a varying number of power levels. It reveals that using more than four levels does not
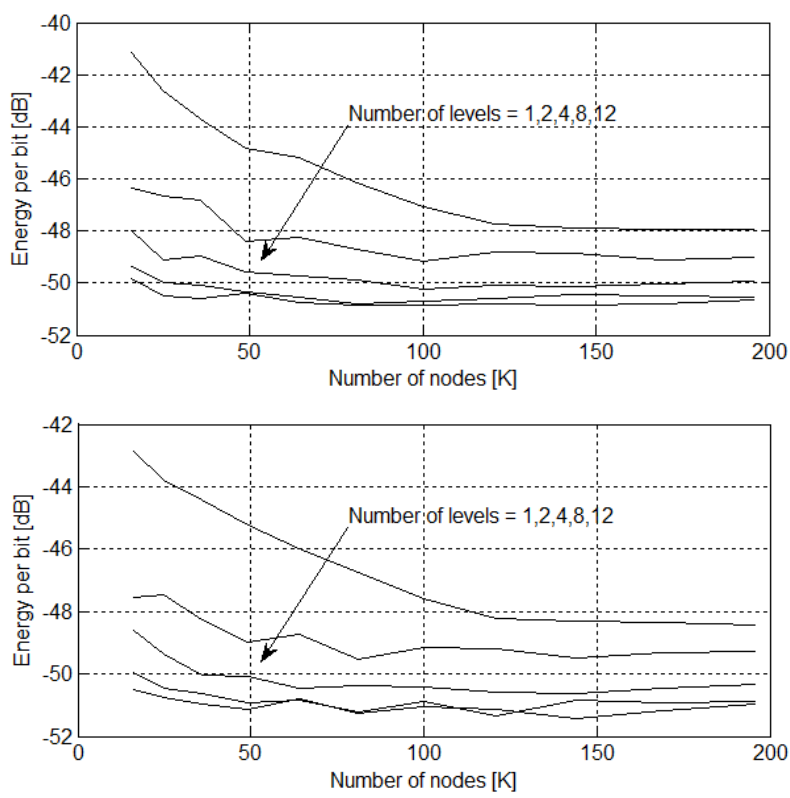

Fig. 8. Energy per bit consumption for CS-ALOHA (top) and DACAP (bottom) for a varying number of power levels.

significantly improve the system performance, which supports the conjecture made earlier in Sec.III-C. Therefore, $N=4$ power levels suffice for the network under consideration as a good compromise between energy per bit consumption and implementation complexity.

\section{Frequency Allocation}

In the previous sections, both $f_{c}$ and $B_{3 d B}$ have been optimized for power consumption according to the channel model used in Sec.II. Here, we illustrate the effect of independently changing these two parameters while using power control with 4 uniformly spaced levels.

\section{1) Center frequency, $f_{c}$}

Fig.9 shows the energy per bit consumption, number of collisions, and average packet end-to-end delay, for the two MAC protocols under study for two choices of center frequency: $f_{c}=30 \mathrm{kHz}$ and $f_{c}=60 \mathrm{kHz}$.

At a higher center frequency, the power consumption for the same inter-node distance is increased because the acoustic path loss is higher. For this reason, the performance is better at $30 \mathrm{kHz}$ than at $60 \mathrm{kHz}$, but this is only so for lower densities. In dense networks, where the transmission distance is small, the energy per bit consumption is nearly the same (Fig.9a).

As the center frequency increases, the interference coming from other nodes also suffers a greater attenuation. Therefore, the total number of collisions with CS-ALOHA can be reduced by increasing the center frequency. This effect can be more clearly seen at high node densities, for which the number of collisions is usually higher (Fig.9b). Because the transmission power scales with the network density, the total energy lost in collisions is small, and the energy performance of CS-ALOHA 

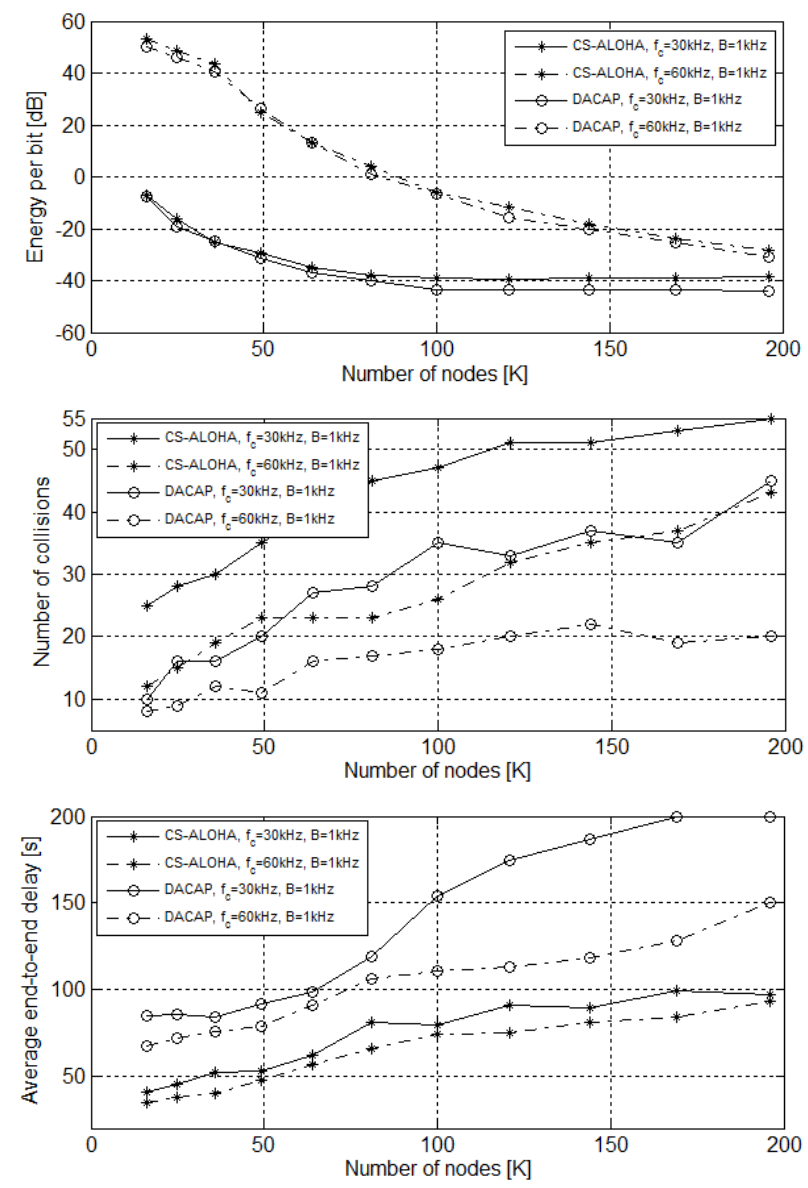

Fig. 9. Energy per bit consumption, number of collisions, and average packet end-to-end delay for CS-ALOHA and DACAP for two choices of center frequency: $f_{c}=30 \mathrm{kHz}$ and $f_{c}=60 \mathrm{kHz} ; \mathrm{N}=4$ power levels.

is close to that of DACAP. At the same time, the end-to-end delay clearly benefits from the reduction in the number of collisions due to a smaller number of retransmissions (Fig.9c). For DACAP, a reduction in interference translates into a reduction in the contention phase duration necessary to avoid collisions.

\section{2) Bandwidth, $B$}

Fig.10 shows the energy per bit consumption, number of collisions, and average packet end-to-end delay for the two MAC protocols under study for two choices of available bandwidth: $B=30 \mathrm{kHz}$ and $B=1 \mathrm{kHz}$.

The energy per bit consumption benefits from a greater bandwidth for two reasons. First, the bit duration $1 / B$ is reduced; thus, the energy per bit reduces too, independently of the MAC protocol used (Fig.10a). Second, packets are shorter and, therefore, less likely to collide (Fig.10b).

This combined effect is what allows a very simple MAC protocol, such as CS-ALOHA, in which collisions are not prevented, to achieve an overall energy per bit performance very close to the more sophisticated collision avoidance
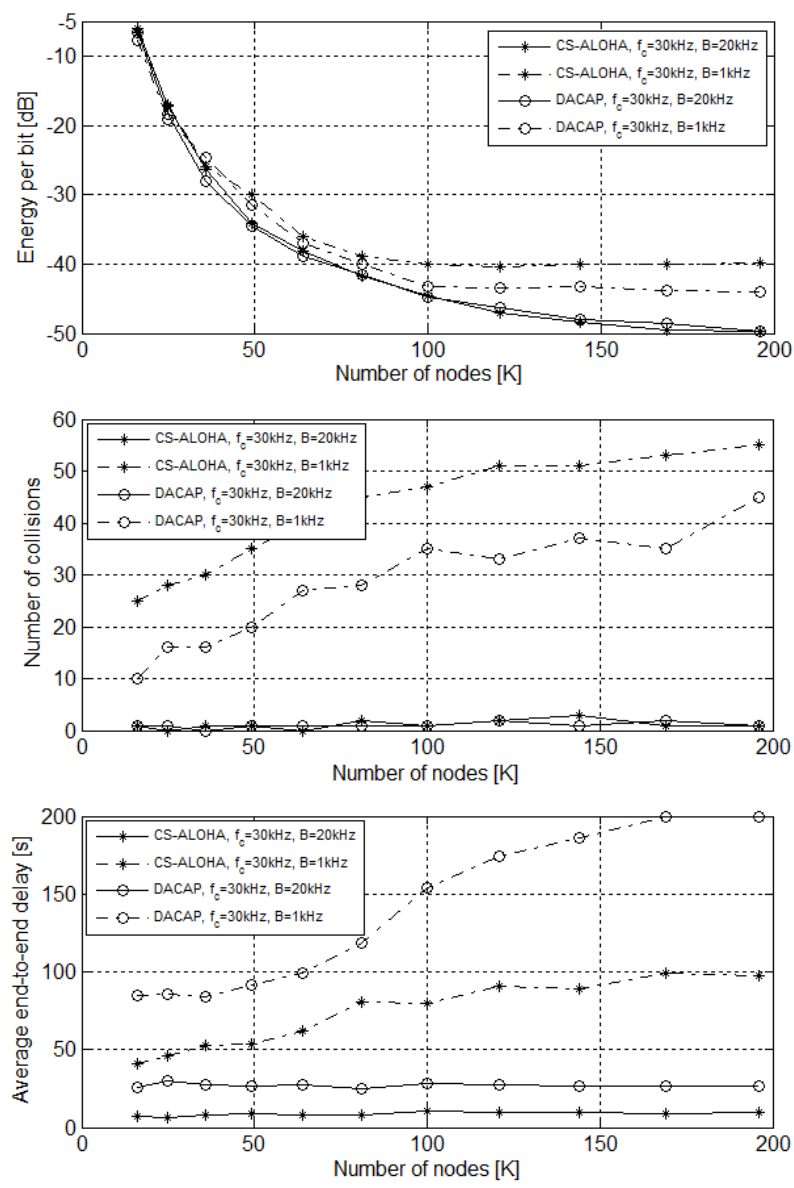

Fig. 10. Energy per bit consumption, number of collisions, and average packet end-to-end delay for CS-ALOHA and DACAP for two choices of bandwidth: $B=30 \mathrm{kHz}$ and $B=1 \mathrm{kHz} ; \mathrm{N}=4$ levels.

DACAP. It also encourages transmission at high bit-rates: even if the application does not require it, the system performance in terms of energy consumption and end-to-end delay will improve.

\section{3) Combined effects, $f_{c}$ and $B$}

When choosing the center frequency and the bandwidth using the minimum power approach introduced in Sec.II, both the optimal center frequency and its corresponding $3 \mathrm{~dB}$ bandwidth increase with the node density. In other words, the average inter-node distance is shorter for a higher node density (Fig.4), and, hence, the optimal frequency and the available bandwidth are higher (Fig.2). As the bandwidth increases, both energy per bit consumption and end-to-end delay are reduced because the packets are shorter and they are less likely to collide.

Fig.11 shows the energy per bit consumption, number of collisions and average packet end-to-end delay for the two MAC protocols under study, for the optimal choice of $f_{c}$ and $B_{3 d B}$. 

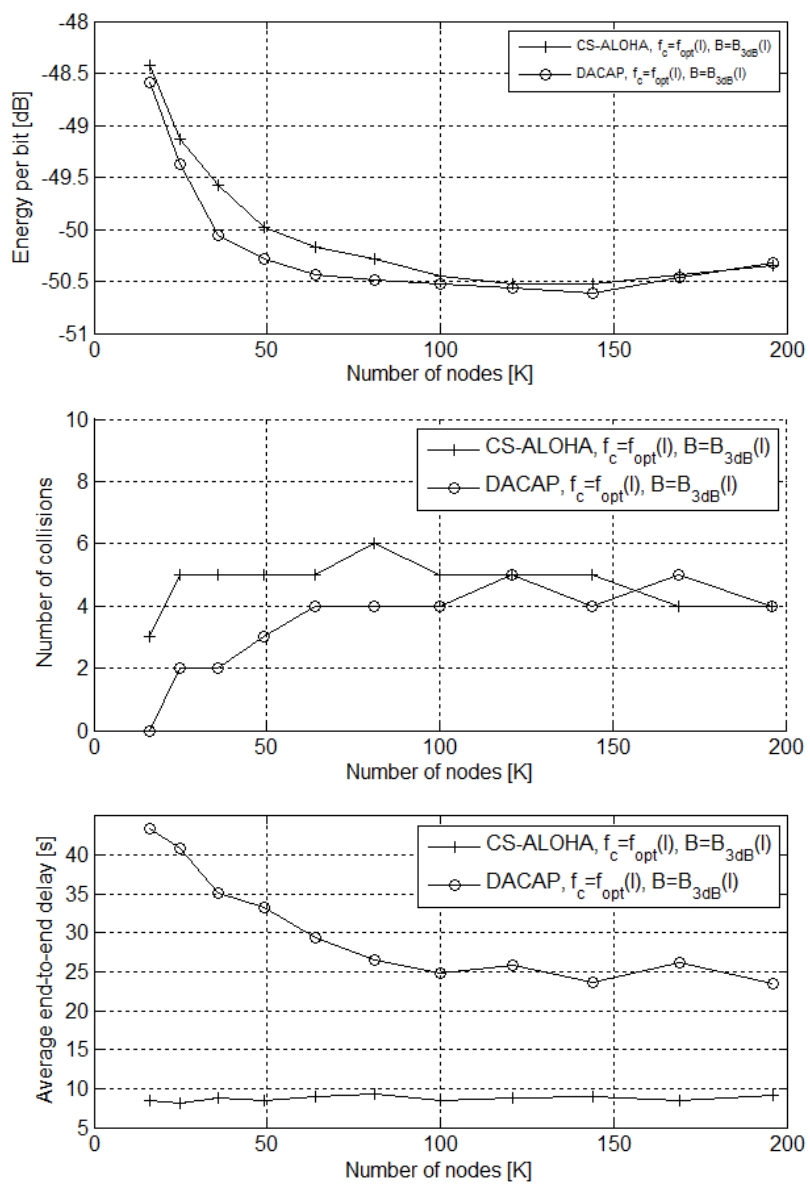

Fig. 11. Energy per bit consumption, number of collisions and average packet end-to-end delay for CS-ALOHA and DACAP for the optimal choice of $f_{c}$ and $B_{3 d B} ; \mathrm{N}=4$ levels.

\section{CONCLUSION}

Discrete power control was considered as a practical means for enabling multi-hop communications for scalable, large coverage in bandwidth-limited underwater acoustic networks. Different power allocation schemes with a varying number of available power levels were considered for varying network densities. For a chosen example scenario, it was shown that four uniformly distributed power levels suffice to achieve energy consumption close to minimum. This number of levels is low enough to motivate a practical implementation of power control.

Due to the dependence of the acoustic path loss on both the distance and the frequency, shorter links are able to utilize higher center frequencies, allowing the system to exploit greater bandwidths. The center frequency and bandwidth were shown to have an effect on both MAC protocols considered. For a higher center frequency, the power required to make up for the greater acoustic path loss is higher, but the interference also attenuates more. With CS-ALOHA, this turns into a reduction in the number of collisions, whereas with DACAP, the waiting time necessary to avoid interfering transmissions becomes shorter, thus effectively reducing the average end-toend delay.

The main benefit however comes from the increase in the available bandwidth. The total energy consumed decreases not only because the transmission time per bit is shorter, but also because shorter packets are less likely to collide. Therefore, the total energy consumed in retransmissions reduces. At the same time, a reduction in the number of collisions implies shorter end-to-end delay. This fact encourages transmission at a high bit-rate: even if the application does not require it, the network performance in terms of energy consumption and end-to-end delay will clearly benefit from it.

Most notably, these effects make CS-ALOHA performance comparable to that of DACAP in terms of energy per bit consumption, and better in terms of average end-to-end delay in high density networks. Hence, by optimizing the frequency allocation, it becomes possible to take full advantage of the simplicity of CS-ALOHA, which is otherwise compromised by channel latency.

\section{ACKNOWLEDGMENT}

This work was supported by the NSF grants 0520075, 0427502 and 0708420, the NOAA Sea Grant NA060AR4170019, and the Neptune Technologies / CEROSCORE-06-02 Grant.

\section{REFERENCES}

[1] M. Stojanovic, "On the Relationship Between Capacity and Distance in an Underwater Acoustic Channel," First ACM International Workshop on Underwater Networks (WUWNet), September 2006.

[2] M. Stojanovic, "Capacity of a Relay Acoustic Link," IEEE Oceans 2007 Conf., pp.1-7, Sept. 29 2007-Oct. 42007.

[3] M. Stojanovic, "On the Design of Underwater Acoustic Cellular Systems,” IEEE Oceans 2007 Conf. - Europe, pp.1-6, 18-21 June 2007.

[4] C.R. Benson, M.J. Ryan, M.R. Frater, "On the Benefits of High Absorption in Practical Multi-hop Networks," IEEE Oceans 2007 Conf. - Europe, pp.1-6, 18-21, June 2007.

[5] A.F. Harris, M. Zorzi, "On the Design of Energy-efficient Routing Protocols in Underwater Networks," 4th Annual IEEE Communications Society Conference on Sensor, Mesh and Ad Hoc Communications and Networks, 2007. SECON '07., pp.80-90, 18-21 June 2007.

[6] J. Gomez, A.T. Campbell, "Variable-Range Transmission Power Control in Wireless Ad Hoc Networks," IEEE Transactions on Mobile Computing, vol.6, no.1, pp.87-99, Jan. 2007.

[7] J.P. Ebert, B. Stremmel, E. Wiederholt, A. Wolisz, "Energy-eficient Power Control Approach for WLANs," Journal of Communications and Networks (JCN), vol. 2, no. 3, pp. 197-206, September 2000.

[8] M. Kubisch, H. Karl, A. Wolisz, L.C. Zhong, J. Rabaey, "Distributed algorithms for transmission power control in wireless sensor networks," 2003 IEEE Wireless Communications and Networking Conf., 2003. WCNC 2003., vol.1, no., pp.558-563 vol.1, 20-20 March 2003.

[9] S. Lin, J. Zhang, G. Zhou, L. Gu, J.A. Stankovic, T. He, "Atpc: adaptive transmission power control for wireless sensor networks," ACM Sensys, 2006.

[10] B. Peleato, M. Stojanovic, "Distance aware collision avoidance protocol for ad-hoc underwater acoustic sensor networks," Communications Letters, IEEE, vol.11, no.12, pp.1025-1027, December 2007.

[11] A. Porto, M. Stojanovic, "Optimizing the Transmission Range in an Underwater Acoustic Network," IEEE Oceans 2007 Conf., pp.1-5, Sept. 29 2007-Oct. 42007.

[12] J.M. Jornet, M. Stojanovic, M. Zorzi, "Focused Beam Routing Protocol for Underwater Acoustic Networks," to appear in WuWNet 08.

[13] J.M. Jornet, "AUVNetSim: A Simulator for Underwater Acoustic Networks", MIT Sea Grant Technical Report, 2008. Available: http://seagrant.mit.edu/media/publications/MITSG08-4.pdf. 